\title{
Diagnosis by axilla skin biopsy in an early case of Lafora's disease
}

\author{
G Rubio, C Garcia Guijo, J J Mallada, A Cabello, A Garcia Merino
}

\begin{abstract}
Myoclonus, seizures and progressive dementia are the main clinical features in Lafora's disease. This is the first reported case in which the diagnosis has been made by axillary skin biopsy in a patient with myoclonus but no other neurological symptoms.
\end{abstract}

(F Neurol Neurosurg Psychiatry 1992;55:1084-1085)

Lafora's disease is a rare autosomal recessive disorder, the presumed metabolic defect of which is unknown. Clinical manifestations are seizures, myoclonus, and progressive dementia. Diagnosis must be established by demonstration of Lafora's inclusion bodies in the biopsy. Carpenter and Karpati ${ }^{1}$ reported in 1981 the diagnostic value of skin biopsy in Lafora's disease; since then, the diagnostic use of this biopsy has been confirmed by other authors. ${ }^{2-9}$ The role of skin biopsy in early stages of the disease, when patients have not yet developed mental deterioration, has not been proved.

We report a young girl with myoclonus affecting the upper limbs and head, and no evidence of dementia, who was diagnosed with Lafora's disease by skin biopsy.

\section{Case report}

A 13 year old girl had been in good health until two years earlier, when she developed occasional myoclonic jerks of the upper limbs and the head. In the preceding three weeks, the frequency of myoclonus had considerably increased. There were no seizures or mental deterioration. Her school performance was normal. Family history showed three siblings who had died from Lafora's disease. Cerebral biopsy confirmed the diagnosis in one of them. A relative of her mother had seizures and three others were mentally retarded. There was no parental consanguinity.

General examination showed no abnormalities. Myoclonic jerks, confined to the head and neck muscles and to the upper limbs, were seen on neurological examination. Alertness, orientation, mood, behaviour and attention were all normal. Tests for recent and remote memory, serial subtraction, and abstract reasoning gave normal results. Language, reading and writing were also normal. There was no apraxia. A Mini Mental State examination showed a score of 30 points. Laboratory findings were unremarkable. EEG showed slow waves, multiple diffuse spikes, polyspikes, and spike-wave discharges with a slow background. CT and MRI scans were normal. Light microscopy of an axilla skin biopsy showed PAS-positive and diastase-resistant intracellular inclusion bodies located in the basal side of the apocrine acini with a striking predominance of the inclusions in the myoepithelial cells in relation to secretory cells (approximately 5:1) (fig 1). Electron microscopy demonstrated round to oval intracytoplasmic inclusions near the nucleus of both the apocrine myoepithelial cells and the apocrine secretory cells (fig 2 ).

Therapy with valproic acid $(600 \mathrm{mg} / \mathrm{d})$ was started and myoclonus disappeared. Three months later, myoclonic jerks returned and absence seizures appeared, requiring an increase in dose. Some days later, she complained of paroxysmal visual hallucinations. Consciousness was not impaired and there were no automatisms. A new EEG showed very frequent high amplitude bilateral paroxysmal discharges with a $2-4 \mathrm{~Hz}$ spike-wave and polyspike pattern. These discharges were accompanied by generalised myoclonic jerks. Photic stimulation provoked $3-\mathrm{Hz}$ polyspikewaves and the patient presented myoclonus involving muscles of the trunk. Background activity was dominated by $4-5 \mathrm{~Hz}$ waves. One year later the patient developed gradually poor school performance and progressive deterioration of her Mini Mental State score fell to 21.

\section{Discussion}

In the proper clinical setting, the presence of Lafora's bodies confirms the diagnosis of Lafora's disease. Detection of Lafora bodies themselves, however, is not diagnostic. Similar polyglucosan inclusions can be seen in normal ageing (corpora amylacea), double athetosis, some instances of amyotrophic lateral sclerosis, type IV glycogen storage disease, and in a group of patients with progressive lower and upper motor neuron deficits, marked sensory loss of the lower extremities, neurogenic bladder, and dementia. ${ }^{7810}$ Only in type IV glycogenosis is the cause of the accumulation of polyglucosan bodies known.

Skin biopsy specimens to confirm the diagnosis of Lafora's disease have been obtained from the calf, forearm, back and axilla. Axillary 


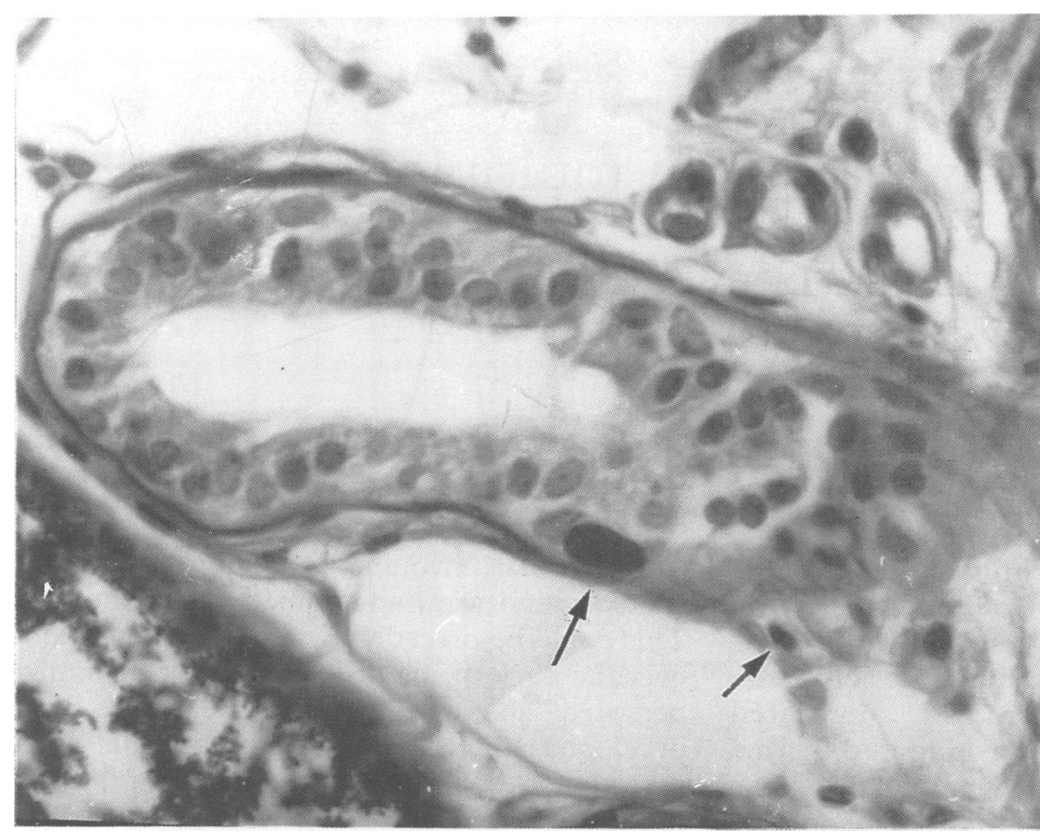

Figure 1 Axillary skin apocrine sweat gland (PAS-diastase $\times 400)$. Oval periodic acid-Schiff (PAS)-positive inclusion bodies are present in the basal side of the acini (arrows) with predominance of the inclusions in the myoepithelial cells.

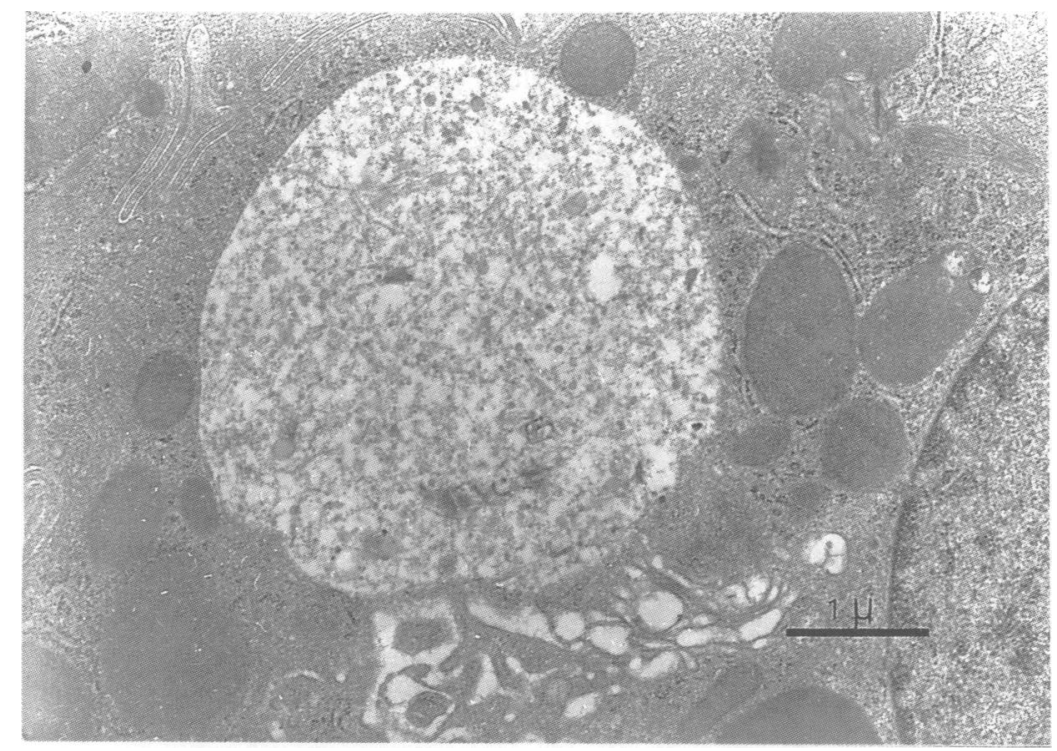

Figure 2 Electron micrograph showing a secretory cell of an apocrine sweat gland containing a round inclusion body without a limiting membrane located near the nucleus. It contains fine granular and filamentous material. basal side of the cells of the eccrine sweat gland ducts. Since then, other authors have described it in the cells of the apocrine sweat gland ducts $^{813}$ and in the acinar cells of the apocrine sweat glands with a predominance of the inclusions in the myoepithelial cells. ${ }^{58}$ In the PAS-reaction these inclusions could be distinguished easily from the normally abundant PAS-positive secretory material by a far more intense lilac-purple staining. ${ }^{5}$

Lafora's bodies are not described in normal subjects, in other pathological states, or in other forms of myoclonus epilepsy; in the appropriate clinical context therefore they may be considered specific for Lafora's disease. Skin biopsy seems to be the most reliable and least aggressive method for demonstrating the presence of Lafora's disease. The PAS-positive inclusion-bodies are sufficiently characteristic to permit diagnosis with light microscopy alone. However, if the nature of the bodies seen on light microscopy is uncertain, a second study by electron microscopy is recommended. If the PAS-stained sections are negative, electron microscopy is not indicated. Furthermore, no abnormalities are found in the axilla skin biopsies of the parents of patients with Lafora's disease. It has not been determined whether typical deposits of Lafora's disease are present in susceptible cells of the siblings of the patients before the appearance of symptoms.

This report shows that Lafora's disease can be diagnosed in the early stages, when only myoclonus is present. This is of practical importance, as skin biopsy may be indicated to classify myoclonus epilepsy when the hereditary pattern is unclear.

1 Carpenter S, Karpati G. Sweat gland duct cells in Lafora disease: Diagnosis by skin biopsy. Neurology 1981;31:1564-8.

2 Roger J, Pellissier JF, Bureau M, et al. Le diagnostic précoce de la maladie de Lafora: importance des manifestations paroxystiques visuelles et intérêt de la biopsie cutanée. Rev Neurol (Paris) 1983;139:115-24.

3 Tinuper P, Aguglia U, Pellissier JF, Gastaut H. Visual ictal phenomena in a case of Lafora disease proven by skin biopsy. Epilepsia 1983;24:214-8.

4 Barbieri F, Santangelo R, Gasparo-Rippa P, Santoro M. Biopsy findings (cerebral cortex, muscle, skin) in Lafora disease. Acta Neurol (Napoli) 1987;9:81-94

5 Busard HLSM, Gabreëls-Festen AAWM, Renier WO, et al. Axilla skin biopsy: a reliable test for the diagnosis of Lafora's disease. Ann Neurol 1987;21:599-601.

6 Newton GA, Sanchez RL, Swedo J, Smith EB. Lafora's disease. The role of skin biopsy. Arch Dermatol 1987;123:1667-9.

7 White Jr JW, Gomez MR. Diagnosis of Lafora disease by skin biopsy. $\mathcal{f}$ Cutan Pathol 1988;15:171-5

8 Samlaska CP, McBurney J, Sau P, James WD. Lafora's disease: What is the best site for skin biopsy. $\mathcal{f}$ Am Acad disease: What is the best site
Dermatol $1989 ; 21(4): 791-2$.

biopsies are preferable because of the higher number of sweat glands whose PAS-positive inclusion bodies can be more easily detected. From data in the literature it seems that PASpositive inclusion bodies of the skin are histologically and histochemically comparable to inclusion bodies of the heart, ${ }^{11} \operatorname{liver}^{1312}$ and CNS; ${ }^{13}$ they only differ ultrastructurally from those found in muscle in lacking a limiting membrane. ${ }^{14}$

Carpenter amd Karpati ${ }^{1}$ described PASpositive inclusion bodies of Lafora's type in the clinicoelectrophysiologic survey of a case of Lafora disease proven by skin biopsy. Epilepsia 1990; 31(2): 194-201.

10 Robitaille Y, Carpenter S, Karpati G, et al. A distinct form of ment of the central and peripheral neuronal processes and astrocytes. Brain 1980;103:315-36.

11 Yokoi S, Aihara Y, Maeda S. The myocardium in Lafora disease. Acta Neuropathol 1975;33:343-9.

12 Nishimura RN, Ishak KG, Reddick R, et al. Lafora disease: diagnosis by liver biopsy. Ann Neurol 1980;8:409-15.

13 Busard BLSM, Renier WO, Gabreëls FJM, et al. Lafora's disease. Comparison of inclusion bodies in skin biopsy and in brain. Arch Neurol 1986;43:296-9.

14 Neville HE, Brooke MH, Austin JH. Studies in myoclonus epilepsy (Lafora body form): IV skeletal muscle abnormalities. Arch Neurol 1974;30:466-74.
9 Kobayashi K, Iyoda K, Ohtsuka Y, et al. Longitudinal adult polyglucosan body disease with massive involve- 